\title{
Improving recovery after elective degenerative spine surgery: 5-year experience with an enhanced recovery after surgery (ERAS) protocol
}

\author{
Victor E. Staartjes, BMed, ${ }^{1,2}$ Marlies P. de Wispelaere, MSc, ${ }^{3}$ and Marc L. Schröder, MD, PhD1 \\ Departments of ${ }^{1}$ Neurosurgery and ${ }^{3}$ Clinical Informatics, Bergman Clinics, Amsterdam, The Netherlands; and ${ }^{2}$ Department of \\ Neurosurgery, Clinical Neuroscience Center, University Hospital Zurich, University of Zurich, Zurich, Switzerland
}

OBJECTIVE Enhanced recovery after surgery (ERAS) has led to a paradigm shift in various surgical specialties. Its application can result in substantial benefits in perioperative healthcare utilization through preoperative physical and mental patient optimization and modulation of the recovery process. Still, ERAS remains relatively new to spine surgery. The authors report their 5-year experience, focusing on ERAS application to a broad population of patients with degenerative spine conditions undergoing elective surgical procedures, including anterior lumbar interbody fusion (ALIF).

METHODS A multimodal ERAS protocol was applied between November 2013 and October 2018. The authors analyze hospital stay, perioperative outcomes, readmissions, and adverse events obtained from a prospective institutional registry. Elective tubular microdiscectomy and mini-open decompression as well as minimally invasive (MI) anterior or posterior fusion cases were included. Their institutional ERAS protocol contains 22 pre-, intra-, and postoperative elements, including preoperative patient counseling, MI techniques, early mobilization and oral intake, minimal postoperative restrictions, and regular audits.

RESULTS A total of 2592 consecutive patients were included, with $199(8 \%)$ undergoing fusion. The mean hospital stay was $1.1 \pm 1.2$ days, with $20(0.8 \%) 30$-day and 36 (1.4\%) 60-day readmissions. Ninety-four percent of patients were discharged after a maximum 1-night hospital stay. Over the 5 -year period, a clear trend toward a higher proportion of patients discharged home after a 1-night stay was observed $(p<0.001)$, with a concomitant decrease in adverse events in the overall cohort $(p=0.025)$ and without increase in readmissions. For fusion procedures, the rate of 1-night hospital stays increased from $26 \%$ to $85 \%$ ( $p<0.001$ ). Similarly, the average length of hospital stay decreased steadily from 2.4 \pm 1.2 days to $1.5 \pm 0.3$ days $(p<0.001)$, with a notable concomitant decrease in variance, resulting in an estimated reduction in nursing costs of $46.8 \%$.

CONCLUSIONS Application of an ERAS protocol over 5 years to a diverse population of patients undergoing surgical procedures, including ALIF, for treatment of degenerative spine conditions was safe and effective, without increase in readmissions. The data from this large case series stress the importance of the multidisciplinary, iterative improvement process to overcome the learning curve associated with ERAS implementation, and the importance of a dedicated perioperative care team. Prospective trials are needed to evaluate spinal ERAS on a higher level of evidence.

https://thejns.org/doi/abs/10.3171/2019.1.FOCUS18646

KEYWORDS elective; degenerative; instrumentation; fusion; enhanced recovery after surgery; ERAS; readmission

$\mathrm{E}$ NHANCED recovery after surgery (ERAS) represents a paradigm shift in surgical patient care and can result in substantial benefits in both clinical outcomes and cost-effectiveness through optimization of the postoperative recovery process. ${ }^{11}$ While the first "fast-track" concepts arose around 1994, ${ }^{8,11}$ the concept of ERAS was first formally introduced in 2001 by the ERAS Study Group, ${ }^{4}$ focusing on both speed and quality of recovery. In 2009, the first guideline was published for colonic and rectal resections. ${ }^{9}$

Protocols aimed at improving recovery after surgery implement multimodal, evidence-based interventions, including but not limited to patient education, minimally invasive (MI) approaches instead of large incisions, careful fluid

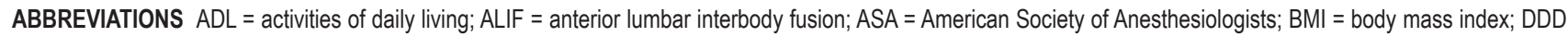
= degenerative disc disease; ERAS = enhanced recovery after surgery; FAQs = frequently asked questions; ICU = intensive care unit; $\mathrm{MI}=$ minimally invasive; $\mathrm{NSAID} \mathrm{=}$ nonsteroidal anti-inflammatory drug; ODI = Oswestry Disability Index; PLIF = posterior lumbar interbody fusion; PROM = patient-reported outcome measure; TLIF = transforaminal lumbar interbody fusion; VAS = visual analog scale.

SUBMITTED November 30, 2018. ACCEPTED January 29, 2019.

INCLUDE WHEN CITING DOI: 10.3171/2019.1.FOCUS18646. 
management, early mobilization, evidence-based nutrition, and regular feedback., ${ }^{5,11,27}$ These interventions can only be achieved within a multidisciplinary framework. For a range of surgical disciplines, there is published evidence highlighting decreases in length of stay, complications, and postoperative symptoms that ERAS can lead to. ${ }^{7,11}$

Currently, major efforts are being conducted to transfer the lessons learned from other surgical specialties and incorporate ERAS protocols into postoperative care of patients undergoing spine surgery. ERAS protocols for posterior lumbar fusion ${ }^{22}$ - even in an endoscopic approach under local anesthesia ${ }^{2,23-25}$ - as well as for decompression, ${ }^{17}$ discectomy, ${ }^{17}$ and spinal oncology ${ }^{6}$ have been described, and prospective studies are ongoing. ${ }^{1}$ However, this field is still in its beginnings, and there is a lack of prospective high-quality data from larger cohorts on interventions for improved recovery after surgery. In particular, we are unaware of published ERAS evidence on anterior lumbar fusion procedures. Moreover, still little is known on the application of such protocols in specialized outpatient or short-stay settings. ${ }^{24,25}$ This applies particularly to implementation of ERAS principles in a broad population of patients undergoing surgical procedures for degenerative spine conditions as seen clinically by neurosurgeons, as opposed to a group of patients all treated with the same single surgical procedure.

Since the inception of the neurosurgical spine unit at Bergman Clinics, a major focus has been put on streamlining the postsurgical recovery and preoperative counseling process, within a self-developed multimodal framework not dissimilar to published ERAS protocols for other surgical specialties. ${ }^{11,24}$ Therefore, the aim of this study is to report the results of our 5-year experience with these measures for improved recovery and to identify any trends potentially related to their implementation.

\section{Methods \\ Overview}

Using data from a prospective single-institutional registry, we identified all patients who underwent elective spine surgery between November 2013 and October 2018 by the senior author (M.L.S.) at a Dutch specialized spine center, Bergman Clinics. During the study period, a multistep protocol was applied to improve rehabilitation after surgery in a multidisciplinary approach. All patients included in this study provided written informed consent for use of their data in studies. The prospective registry received approval by the local institutional review board (Medical Research Ethics Committees United), and this study was performed according to the Declaration of Helsinki.

\section{Implementation of ERAS}

The elective spine program at our center has from its inception been laid out to enable highly efficient treatment of patients with degenerative spinal pathologies, with a focus on minimal hospital stay, optimization of subjective patient recovery, use of modern, minimally invasive (MI) operative techniques, and regular patient feedback, in a multidisciplinary approach. On November 1, 2013, the final element of the currently reported ERAS protocol was introduced: Automatically dispatched digital assessment of patient-reported outcome measures (PROMs) for enhanced monitoring. ${ }^{14}$ The data reported in this study thus represent our 5-year institutional experience. A summary of the protocol is provided in Table 1 . To be included in this study, patients needed to be operated on since implementation of the current study protocol and undergo surgery for lumbar disc herniation, spinal stenosis, spondylolisthesis, facet cysts, or proven degenerative disc disease (DDD), with a minimum follow-up of 30 days to assess readmissions. ${ }^{19}$

\section{Anesthesiologic Screening}

Patients who were older than 80 years or had a body mass index (BMI) greater than $33 \mathrm{~kg} / \mathrm{m}^{2}$ or an American Society of Anesthesiologists (ASA) score greater than 2 were not eligible for surgery in this short-stay setting, as dictated by local insurance policy. Patients with a BMI greater than $30 \mathrm{~kg} / \mathrm{m}^{2}$ received structured nutritional advice and counseling and were required to lose sufficient weight before being considered for surgery. Severe sleep apnea syndrome was also a contraindication for surgery. Patients on blood thinners are never considered for surgery in our setting. This is due to the often-associated comorbidities and the risk of major bleeding, which represent a high surgical risk in a setting without an intensive care unit (ICU). However, patients who were treated solely with acetylsalicylic acid were considered for nonfusion procedures and were told not to stop taking their medication perioperatively. Strict blood pressure regulation was maintained. Patients with hypertension at the preoperative screening were required to consult with their general practitioner or cardiologist to regulate their blood pressure before being considered for surgery. Proven osteoporosis was a contraindication for fusion procedures at our center. Patients who presented with a higher risk profile than delineated by our screening thresholds were always referred to larger, academic, or community hospitals.

\section{Preoperative Patient Counseling}

All patients were strongly advised to cease tobacco and alcohol consumption preoperatively, and patients eligible for fusion surgery were required to cease smoking at least 3 months before being considered for surgical treatment. All patients were systematically educated on what to expect during their recovery period. We provided all patients with the same three principles of conduct ("Three Golden Rules") during the recovery process, namely: 1) You are allowed to do anything you want, but 2) you must listen to your body carefully, and 3) you must stop what you are doing when you experience too much pain. Furthermore, patients were asked to cease working and work-related activities at home for the first 3 postoperative weeks. This was worded as an "investment in their recovery process." Patients were advised not to drive a car within 3 weeks of surgery, because of potential liability. Patients were instructed not to undergo physical therapy during the first 3 months. ${ }^{13}$ No restrictions on activities of daily living (ADL) were set. A patient-friendly website that includes a range of frequently asked questions (FAQs) and detailed 
TABLE 1. Elements of institutional protocol for improving recovery after surgery

\begin{tabular}{|c|c|c|c|}
\hline Time Point & Element & Summary & Discipline \\
\hline \multicolumn{4}{|l|}{ Preop } \\
\hline 1 & $\begin{array}{l}\text { Control of smoking \& alcohol } \\
\text { intake }\end{array}$ & $\begin{array}{l}\text { Advised to cease smoking \& alcohol intake before op; cessation of smoking } 3 \\
\text { mos before fusion op }\end{array}$ & Neurosurgery \\
\hline 2 & Strict patient screening & $\begin{array}{l}\text { Strict anesthesiologic screening \& patient selection to enhance periop patient } \\
\text { safety w/ targeted optimization of comorbidities }\end{array}$ & Anesthesiology \\
\hline 3 & Weight loss in obese patients & Structured nutritional advice \& counseling for patients $w /$ a BMI $>30 \mathrm{~kg} / \mathrm{m}^{2}$ & Multidisciplinary \\
\hline 4 & Patient education & $\begin{array}{l}\text { Systematic education on what to expect during recovery; } 3 \text { simple principles } \\
\text { of conduct provided ("Three Golden Rules") }\end{array}$ & Neurosurgery \\
\hline 5 & Prophylaxis against infection & Prophylactic use of a broad-spectrum antibiotic & Anesthesiology \\
\hline 6 & Prophylaxis against thrombosis & Prophylactic use of low-molecular-weight heparin & Anesthesiology \\
\hline \multicolumn{4}{|l|}{ Intraop } \\
\hline 7 & $\begin{array}{l}\text { Standardized anesthesia \& avoid- } \\
\text { ance of long-acting opioids }\end{array}$ & General anesthesia maintained using propofol \& a short-acting opioid & Anesthesiology \\
\hline 8 & Local analgesia & Infiltration of surgical site w/ local analgesic agents & Neurosurgery \\
\hline 9 & MI surgical techniques & $\begin{array}{l}\text { Use of tubular working channels, robotic guidance, \& MI or mini-open ap- } \\
\text { proaches, avoiding large incisions \& associated muscle damage }\end{array}$ & Neurosurgery \\
\hline 10 & Limited use of muscle relaxants & $\begin{array}{l}\text { Sparing use of muscle relaxants, enabling more efficient mobilization \& } \\
\text { recovery }\end{array}$ & Anesthesiology \\
\hline 11 & $\begin{array}{l}\text { Prevention of fluid imbalance \& } \\
\quad \text { blood transfusion }\end{array}$ & $\begin{array}{l}\text { Minimization of over- or underhydration; administration of vasopressors to } \\
\text { regulate BP; availability of autologous cell-salvage transfusion during all } \\
\text { procedures }\end{array}$ & Anesthesiology \\
\hline 12 & Prevention of hypothermia & Control of body temperature by means of warm-air blankets & Anesthesiology \\
\hline \multicolumn{4}{|l|}{ Postop } \\
\hline 13 & $\begin{array}{l}\text { Sparing use \& early removal of } \\
\text { surgical site drains \& urinary } \\
\text { catheters }\end{array}$ & $\begin{array}{l}\text { Use of surgical site drains only used after mini-open decompression or MI- } \\
\text { PLIF; removal of drains \& catheters as early as possible }\end{array}$ & Multidisciplinary \\
\hline 14 & Opioid-sparing analgesia & $\begin{array}{l}\text { Effective analgesia achieved using NSAIDs \& paracetamol; patient-controlled } \\
\text { analgesia w/ short-acting opioids avoided }\end{array}$ & Multidisciplinary \\
\hline 15 & Early mobilization & $\begin{array}{l}\text { Whenever feasible, mobilization } 2 \text { hrs after op under guidance of a physical } \\
\text { therapist }\end{array}$ & Physical therapy \\
\hline 16 & Early intake of solids \& fluids & Encouragement of oral intake of solids \& fluids at will on the day of op & Multidisciplinary \\
\hline 17 & Preparation for early discharge & $\begin{array}{l}\text { Integration of relatives \& early organization of transport, allowing patients to } \\
\text { be discharged home early after a minimum 1-night stay }\end{array}$ & Multidisciplinary \\
\hline \multicolumn{4}{|c|}{ Post-discharge } \\
\hline 18 & Minimal restriction of $A D L$ & No restrictions set w/ respect to $A D L$ & Neurosurgery \\
\hline 19 & Patient-friendly website w/ FAQs & $\begin{array}{l}\text { Patients provided } w / \text { a website that includes a range of FAQs as well as } \\
\text { detailed information on the recovery process }\end{array}$ & Multidisciplinary \\
\hline 20 & Scheduled early FU by phone & $\begin{array}{l}\text { Patients telephoned } 2 \text { days } \& 2 \text { wks after op to check on status of their } \\
\text { recovery process }\end{array}$ & Neurosurgery \\
\hline 21 & $\begin{array}{l}\text { Low threshold for clinical FU visit/ } \\
\text { readmission }\end{array}$ & $\begin{array}{l}\text { Patients instructed to call in } 24 / 7 \mathrm{w} / \text { any uncertainties; establishment of low } \\
\text { threshold for clinical FU visit or readmission, if desired by the patient }\end{array}$ & Multidisciplinary \\
\hline 22 & Regular digital audit/FU & $\begin{array}{l}\text { Questionnaires automatically sent to patients digitally at } 6 \text { wks, } 12 \text { mos, \& } 24 \\
\text { mos, allowing for effective FU }\end{array}$ & Multidisciplinary \\
\hline
\end{tabular}

$\mathrm{BP}=$ blood pressure; $\mathrm{FU}=$ follow-up; $24 / 7=24$ hours a day, 7 days a week.

information on the recovery process has been set up and is frequently visited by patients who have questions postoperatively. Furthermore, patients have a scheduled telephone call 2 days and 14 days after surgery to check on their status. Only fusion patients had a scheduled early 6-week clinical and radiological follow-up. Patients were instructed to call in 24/7 (24 hours a day, 7 days a week) with any unanswered questions, and were informed that, if necessary or desired by the patient, there was a low threshold for a clinical follow-up visit or readmission.

\section{Surgery and Perioperative Management}

Patients underwent MI tubular microdiscectomy, single-level robot-guided MI posterior lumbar interbody fusion (PLIF) or transforaminal lumbar interbody fusion (TLIF), mini-open anterior lumbar interbody fusion 


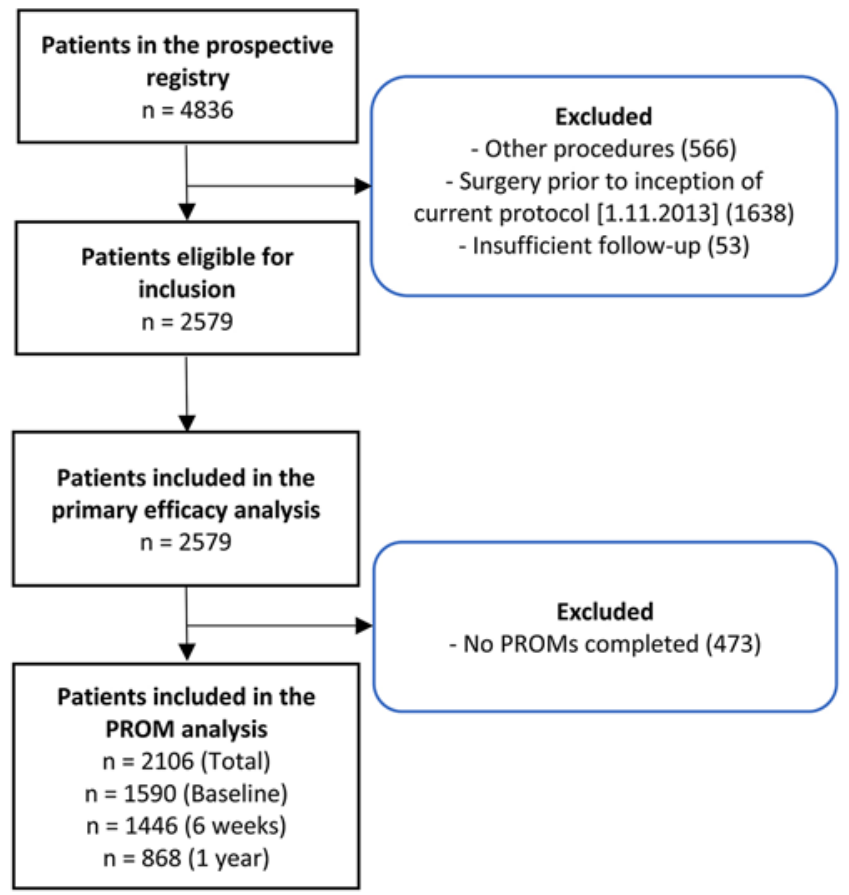

FIG. 1. Flowchart demonstrating the flow of patients in the prospective registry throughout this analysis.

(ALIF), or mini-open decompression, as described previously. $3,15,17,19$ Preoperatively, patients received cefazolin as antibiotic prophylaxis and low-molecular-weight heparin as prophylaxis against thrombosis. For local analgesia, 2.5 $\mathrm{mg} / \mathrm{ml}$ of ropivacaine was intramuscularly administered prior to incision. General anesthesia was maintained using propofol and a short-acting opioid (sufentanil). The use of muscle relaxants was limited to allow for faster recovery. Fluid imbalance was minimized, vasopressors were administered to regulate blood pressure, and autologous cell-salvage transfusion was available during all procedures. Surgical site drains were used only for mini-open decompression and MI-PLIF and were removed as soon as possible. Urinary catheters were removed early. Postoperatively, opioid-sparing analgesia was maintained and adjusted as appropriate using nonsteroidal anti-inflammatory drugs (NSAIDs) and paracetamol. We considered using patient-controlled analgesia with intravenous piritramide and long-acting opioids only for patients with unmanageable pain after fusion procedures. Whenever feasible, patients were mobilized 2 hours after surgery under guidance of a physical therapist and were discharged home after a minimum stay of 1 night, as soon as the following conditions were true: 1) pain controlled by oral analgesics, 2) no complication (e.g., incidental durotomy) that would require prolonged hospital stay, and 3) ability to climb stairs and to perform ADL. Fusion patients were provided with a light elastic brace.

\section{Data Collection and Statistical Analysis}

Before the first visit, all patients received an invitation to complete an online baseline questionnaire on a validated Web-based application. ${ }^{14}$ At 6 weeks, 12 months, and 24 months after surgery, scheduled follow-up questionnaires were automatically sent out to all patients digitally and completed in the same fashion. All adverse events were systematically collected in a separate database, and reoperations as well as 30-day and 60-day readmissions were tracked. Continuous variables are expressed as medians and interquartile ranges and means \pm standard deviations, and categorical variables are expressed as numbers (percentages). Paired and unpaired observations were assessed using the exact versions of Wilcoxon's signed-rank and rank-sum tests, respectively, based on the "shift" algorithm described by Streitberg and Röhmel. ${ }^{20}$ Trends over the years were statistically tested for. The Cochrane-Armitage test was applied for dichotomous variables, and the exact version of the Jonckheere-Terpstra test, based on 10,000 permutations, was applied for continuous variables. A single-subgroup analysis was performed to assess trends in hospital stay for pooled fusion procedures. Data from November and December of 2013 and 2014 were pooled in a single group (2013-2014) to achieve an approximately equal sample size distribution among the years analyzed. All analyses were carried out using $\mathrm{R}$ version 3.5.1 (The $\mathrm{R}$ Foundation for Statistical Computing). A 2-tailed $p \leq 0.05$ was considered statistically significant. The statistical code ( $\mathrm{R}$ code) is provided as Online-Only Content.

\section{Results}

A total of 2592 consecutive patients were included in the primary efficacy analysis (Fig. 1). Of these, 2117 (82\%) completed at least 1 PROM invitation. Detailed patient characteristics are provided in Table 2.

\section{Perioperative Results}

Hospital Stay

On average, we recorded a hospital stay of $1.1 \pm 1.2$ days (Table 3). ALIF patients were discharged after $1.4 \pm 0.7$ days, and MI-TLIF patients after $1.9 \pm 0.6$ days $(\mathrm{p}<0.001)$. The proportion of patients discharged on the day of surgery or on the day after surgery was $94 \%$ (2425 patients), with the highest rate among discectomy patients (98\%), and the lowest rate for MI-PLIF patients (22\%); $85 \%$ of ALIF and $52 \%$ of MI-TLIF patients were discharged after a 1-night stay. For patients who were discharged on the day after surgery, the mean time of day at discharge was 10:49 $\mathrm{AM} \pm 2: 54$ hours.

\section{Readmissions, Adverse Events, and Reoperations}

The rate of 30-day readmissions was $0.78 \%$ (20 patients). Similarly, 36 patients $(1.40 \%)$ were readmitted within 60 days. The most frequent reasons for readmission were unmanageable pain (67\%) and persistent CSF leakage presenting with dizziness and orthostatic headache (17\%). A detailed report of readmissions is provided in Supplementary Table 1. Adverse events were recorded in 95 patients (4\%, Supplementary Table 2). Overall, 200 patients $(8 \%)$ required reoperation (Supplementary Table 3), including $151(6 \%)$ who required reoperation at the index level.

\section{Patient-Reported Outcome Measures}

All PROMs were vastly improved from the baseline 
TABLE 2. Summary of baseline patient characteristics

\begin{tabular}{|c|c|c|c|c|c|c|}
\hline Parameter & Overall & ALIF & MI-TLIF & MI-PLIF & Discectomy & Decompression \\
\hline No. of patients (\%) & $2579(100)$ & $61(2)$ & $87(3)$ & $51(2)$ & $1929(75)$ & $451(17)$ \\
\hline Age in yrs, mean & $48.5 \pm 13.5$ & $44.1 \pm 8.1$ & $49.2 \pm 11.8$ & $50.5 \pm 12.7$ & $45.3 \pm 12.4$ & $62.5 \pm 9.7$ \\
\hline Female sex, n (\%) & $1184(46)$ & $40(66)$ & $38(44)$ & $26(51)$ & $865(45)$ & $215(48)$ \\
\hline $\mathrm{BMI}$ in $\mathrm{kg} / \mathrm{m}^{2}$, mean & $25.5 \pm 3.3$ & $24.8 \pm 2.8$ & $25.8 \pm 3.2$ & $26.5 \pm 3.5$ & $25.4 \pm 3.3$ & $26.1 \pm 3.2$ \\
\hline Active smoker, n (\%) & $684(27)$ & $15(25)$ & $22(25)$ & $13(25)$ & $531(28)$ & $103(23)$ \\
\hline Prior op, n (\%) & $286(11)$ & $14(23)$ & $13(15)$ & $10(20)$ & $206(11)$ & $43(10)$ \\
\hline \multicolumn{7}{|l|}{ ASA score, $n(\%)$} \\
\hline 1 & $1539(60)$ & $42(69)$ & $55(63)$ & $23(45)$ & $1244(64)$ & $175(39)$ \\
\hline$\|$ & $1032(40)$ & $19(31)$ & $32(37)$ & $28(55)$ & $679(35)$ & $274(61)$ \\
\hline III & $8(0)$ & $0(0)$ & $0(0)$ & $0(0)$ & $6(1)$ & $2(0)$ \\
\hline \multicolumn{7}{|l|}{ Index level, n (\%) } \\
\hline L1-2 & $1(0)$ & $0(0)$ & $0(0)$ & $0(0)$ & $0(0)$ & $1(0)$ \\
\hline L2-3 & $82(3)$ & $0(0)$ & $0(0)$ & $0(0)$ & $24(1)$ & $58(13)$ \\
\hline L3-4 & $285(11)$ & $2(3)$ & $3(3)$ & $2(4)$ & $127(7)$ & $151(33)$ \\
\hline L4-5 & $1156(45)$ & $26(43)$ & $39(45)$ & $19(37)$ & $845(44)$ & $227(50)$ \\
\hline L5-S1 & $1055(41)$ & $33(54)$ & $45(52)$ & $30(59)$ & $933(48)$ & $14(3)$ \\
\hline \multicolumn{7}{|l|}{ Indication for op, n (\%) } \\
\hline Lumbar disc herniation & $1911(74)$ & $0(0)$ & $0(0)$ & $0(0)$ & $1911(99)$ & $0(0)$ \\
\hline Lumbar spinal stenosis & $451(17)$ & $0(0)$ & $0(0)$ & $0(0)$ & $0(0)$ & $451(100)$ \\
\hline DDD & $101(4)$ & $61(100)$ & $28(32)$ & $12(24)$ & $0(0)$ & $0(0)$ \\
\hline Spondylolisthesis & $98(4)$ & $0(0)$ & $59(68)$ & $39(76)$ & $0(0)$ & $0(0)$ \\
\hline Facet joint cyst & $18(1)$ & $0(0)$ & $0(0)$ & $0(0)$ & $18(1)$ & $0(0)$ \\
\hline \multicolumn{7}{|l|}{$\mathrm{Yr}, \mathrm{n}(\%)$} \\
\hline 2013-2014 & $588(100)$ & $17(3)$ & $10(2)$ & $34(6)$ & $421(72)$ & $106(18)$ \\
\hline 2015 & $576(100)$ & $21(4)$ & $19(3)$ & $8(1)$ & $426(74)$ & $102(18)$ \\
\hline 2016 & $518(100)$ & $10(2)$ & $22(4)$ & $0(0)$ & $396(76)$ & $90(17)$ \\
\hline 2017 & $480(100)$ & $5(1)$ & $22(5)$ & $4(1)$ & $371(77)$ & $78(16)$ \\
\hline 2018 & $417(100)$ & $8(2)$ & $14(3)$ & $5(1)$ & $315(76)$ & $75(18)$ \\
\hline \multicolumn{7}{|l|}{ Baseline PROMs, mean } \\
\hline NRS back pain severity & $5.5 \pm 2.8$ & $6.4 \pm 2.1$ & $6.8 \pm 2.2$ & $6.4 \pm 2.8$ & $5.4 \pm 2.8$ & $5.4 \pm 3.0$ \\
\hline NRS leg pain severity & $7.2 \pm 2.3$ & $5.9 \pm 2.8$ & $6.0 \pm 2.8$ & $6.2 \pm 3.0$ & $7.5 \pm 2.0$ & $6.6 \pm 2.6$ \\
\hline ODI & $47.0 \pm 17.8$ & $48.7 \pm 13.5$ & $39.4 \pm 15.0$ & $43.5 \pm 19.3$ & $49.0 \pm 17.8$ & $40.0 \pm 16.5$ \\
\hline EQ-5D index & $0.40 \pm 0.31$ & $0.56 \pm 0.28$ & $0.52 \pm 0.28$ & $0.63 \pm 0.20$ & $0.37 \pm 0.31$ & $0.49 \pm 0.30$ \\
\hline EQ-VAS & $50.8 \pm 18.4$ & $64.9 \pm 20.8$ & $57.1 \pm 13.8$ & $63.4 \pm 15.2$ & $49.1 \pm 18.2$ & $55.4 \pm 18.7$ \\
\hline
\end{tabular}

NRS = numeric rating scale.

Mean values are presented with standard deviations.

measurement (Table 2) at the 6-week (Table 3) follow-up (all $\mathrm{p}<0.001$ ). From the 6 -week to the 1-year follow-up, however, we observed significant further improvement of functional impairment ( $\Delta$ Oswestry Disability Index [ODI]: $-7.8 \pm 17.0, \mathrm{p}<0.001)$ and in health-related quality of life in terms of EQ-5D index $(\Delta: 0.08 \pm 0.23$, p < $0.001)$ and EQ-VAS (visual analog scale) $(\Delta: 4.0 \pm 16.8$, p $=0.005)$, while neither leg pain $(\mathrm{p}=0.394)$ nor back pain $(\mathrm{p}=0.948)$ severity were further reduced.

\section{Trend Analysis}

Overall

The decreasing trend in length of surgery (Table 4) from $38.8 \pm 36.1$ minutes in $2013-2014$ to $29.0 \pm 22.8$ minutes in 2018 was statistically significant $(\mathrm{p}<0.001)$. The apparent reduction in length of hospital stay from $29.8 \pm 17.8$ hours to $26.8 \pm 9.2$ hours during the 5 -year experience did not reach significance $(p=0.266)$. However, the proportion of patients who were discharged the morning after surgery demonstrated a steady, increasing, significant trend from $90 \%$ to $96 \%$ ( $\mathrm{p}<0.001$ ). There was no significant change in 30 -day $(\mathrm{p}=0.087$ ) or 60 -day ( $\mathrm{p}$ $=0.073$ ) readmissions over time. Adverse events demonstrated a decreasing trend $(\mathrm{p}=0.025)$.

\section{Fusion Procedures}

All lumbar fusion procedures $(n=199)$ were pooled for a selective subgroup analysis (Table 5). In this population, 
TABLE 3. Summary of perioperative results and adverse events

\begin{tabular}{|c|c|c|c|c|c|c|}
\hline Parameter & Overall & ALIF & MI-TLIF & MI-PLIF & Discectomy & Decompression \\
\hline No. of patients (\%) & $2579(100)$ & $61(2)$ & $87(3)$ & $51(2)$ & $1929(75)$ & $451(17)$ \\
\hline Length of op in mins, mean & $32.2 \pm 29.7$ & $79.1 \pm 20.0$ & $126.3 \pm 46.6$ & $141.3 \pm 47.6$ & $24.8 \pm 10.7$ & $26.8 \pm 14.0$ \\
\hline Length of hospital stay in days, mean & $1.1 \pm 1.2$ & $1.4 \pm 0.7$ & $1.9 \pm 0.6$ & $2.4 \pm 1.2$ & $1.0 \pm 0.8$ & $1.2 \pm 0.6$ \\
\hline Length of hospital stay in hrs, mean & $26.9 \pm 18.9$ & $34.4 \pm 15.6$ & $45.3 \pm 14.1$ & $58.6 \pm 28.4$ & $24.8 \pm 18.4$ & $27.8 \pm 15.5$ \\
\hline 1-night hospital stay, n (\%) & $2425(94)$ & $52(85)$ & $45(52)$ & $11(22)$ & $1882(98)$ & $435(96)$ \\
\hline \multicolumn{7}{|l|}{ Readmission rate, $\mathrm{n}(\%)$} \\
\hline 30-day readmissions & $20(1)$ & $1(2)$ & $1(1)$ & $3(6)$ & $12(1)$ & $3(1)$ \\
\hline 60-day readmissions & $36(1)$ & $2(3)$ & $1(1)$ & $3(6)$ & $26(1)$ & $4(1)$ \\
\hline Adverse events, $n(\%)$ & $95(4)$ & $2(3)$ & $7(8)$ & $6(12)$ & $60(3)$ & $20(4)$ \\
\hline Reoperation rate at index level, $\mathrm{n}(\%)$ & $151(6)$ & $1(2)$ & $0(0)$ & $1(2)$ & $121(6)$ & $28(6)$ \\
\hline Overall reoperation rate, $\mathrm{n}(\%)$ & $200(8)$ & $4(7)$ & $4(5)$ & $2(4)$ & $150(8)$ & $40(9)$ \\
\hline \multicolumn{7}{|l|}{ Short-term PROMs (6 wks), mean } \\
\hline NRS back pain severity & $3.2 \pm 2.5$ & $3.6 \pm 2.3$ & $3.7 \pm 2.5$ & $2.9 \pm 2.1$ & $3.3 \pm 2.5$ & $2.5 \pm 2.3$ \\
\hline NRS leg pain severity & $2.2 \pm 2.6$ & $3.0 \pm 2.3$ & $2.2 \pm 2.5$ & $2.1 \pm 2.4$ & $2.1 \pm 2.6$ & $2.6 \pm 2.8$ \\
\hline ODI & $23.8 \pm 17.5$ & $29.7 \pm 17.2$ & $31.5 \pm 19.0$ & $22.5 \pm 17.3$ & $23.7 \pm 17.3$ & $22.4 \pm 17.7$ \\
\hline EQ-5D index & $0.72 \pm 0.24$ & $0.79 \pm 0.12$ & $0.61 \pm 0.29$ & $0.60 \pm 0.22$ & $0.72 \pm 0.24$ & $0.75 \pm 0.23$ \\
\hline EQ-VAS & $68.7 \pm 16.7$ & $78.3 \pm 11.3$ & $65.0 \pm 18.3$ & $69.9 \pm 12.3$ & $68.0 \pm 16.6$ & $71.4 \pm 16.9$ \\
\hline \multicolumn{7}{|l|}{ Long-term PROMs (1 yr), mean } \\
\hline NRS back pain severity & $3.1 \pm 2.7$ & $3.6 \pm 2.9$ & $4.1 \pm 2.8$ & $3.4 \pm 2.8$ & $3.1 \pm 2.7$ & $2.6 \pm 2.7$ \\
\hline NRS leg pain severity & $2.2 \pm 2.7$ & $3.0 \pm 2.8$ & $2.1 \pm 2.5$ & $1.7 \pm 2.4$ & $2.1 \pm 2.7$ & $2.5 \pm 3.0$ \\
\hline ODI & $16.0 \pm 16.4$ & $25.0 \pm 20.1$ & $18.6 \pm 16.5$ & $20.2 \pm 19.3$ & $15.1 \pm 15.8$ & $16.3 \pm 17.1$ \\
\hline EQ-5D index & $0.80 \pm 0.22$ & $0.73 \pm 0.24$ & $0.79 \pm 0.24$ & $0.84 \pm 0.03$ & $0.79 \pm 0.22$ & $0.81 \pm 0.22$ \\
\hline EQ-VAS & $72.7 \pm 16.9$ & $69.8 \pm 22.7$ & $71.2 \pm 20.7$ & $72.3 \pm 20.1$ & $73.5 \pm 14.6$ & $71.0 \pm 22.0$ \\
\hline
\end{tabular}

Mean values are presented with standard deviations.

the rate of 1-night hospital stays demonstrated a marked and steady increase (Fig. 2), from 26\% in 2013-2014 to $85 \%$ in 2018 ( $\mathrm{p}<0.001)$. Similarly, the average length of hospital stay (Fig. 3) decreased steadily, from 2.4 \pm 1.2 days (56.9 \pm 28.6 hours) in 2013-2014 to $1.5 \pm 0.3$ days (34.9 \pm 7.9 hours) in 2018 ( $\mathrm{p}<0.001$ ), with a notable concomitant decrease in variance. Based on this change, our Department of Finance estimates a reduction in nursing costs of $46.8 \%$.

\section{Discussion}

Strategies to improve recovery after surgery, both by optimizing the patient's physical and mental condition preoperatively and by reducing the burden of the procedure and perioperative stay itself, are gaining traction in a wide range of specialties. While the single elements of an ERAS protocol alone may not modulate the incidence of adverse events, patient comfort, and the surgical stress

TABLE 4. Results of the trend analysis

\begin{tabular}{|c|c|c|c|c|c|c|c|c|}
\hline \multirow[b]{2}{*}{ Parameter } & \multicolumn{5}{|c|}{ Value } & \multirow[b]{2}{*}{ Trend } & \multirow[b]{2}{*}{ Effect Size } & \multirow[b]{2}{*}{$\begin{array}{l}p \text { Value } \\
\text { (trend) }\end{array}$} \\
\hline & $\begin{array}{c}2013-2014 \\
(n=588)\end{array}$ & $\begin{array}{c}2015 \\
(n=576)\end{array}$ & $\begin{array}{c}2016 \\
(n=518)\end{array}$ & $\begin{array}{c}2017 \\
(n=480)\end{array}$ & $\begin{array}{c}2018 \\
(n=417)\end{array}$ & & & \\
\hline Length of op in mins, mean & $38.8 \pm 36.1$ & $32.8 \pm 28.0$ & $30.0 \pm 30.1$ & $28.8 \pm 26.8$ & $29.0 \pm 22.8$ & Decreasing & $T_{J T}=1,072,100$ & $<0.001^{*}$ \\
\hline Length of hospital stay in hrs, mean & $29.8 \pm 17.8$ & $25.5 \pm 13.0$ & $24.4 \pm 30.6$ & $27.7 \pm 15.7$ & $26.8 \pm 9.2$ & - & $\mathrm{T}_{\mathrm{JT}}=1,332,500$ & 0.266 \\
\hline 1-night hospital stay, n (\%) & $528(90)$ & $542(94)$ & $493(95)$ & $461(96)$ & $401(96)$ & Increasing & $Z=-4.59$ & $<0.001^{*}$ \\
\hline \multicolumn{9}{|l|}{ Readmission rate, $\mathrm{n}(\%)$} \\
\hline 30-day readmissions & $8(1)$ & $3(1)$ & $6(1)$ & $1(0)$ & $2(0)$ & - & $Z=1.71$ & 0.087 \\
\hline 60 -day readmissions & $11(2)$ & $8(1)$ & $10(2)$ & $5(1)$ & $2(0)$ & - & $Z=1.79$ & 0.073 \\
\hline Adverse events, n (\%) & $30(5)$ & $22(4)$ & $19(4)$ & $12(2)$ & $12(3)$ & Decreasing & $Z=2.24$ & $0.025^{*}$ \\
\hline
\end{tabular}

$\mathrm{T}_{\mathrm{JT}}=$ Jonckheere-Terpstra effect size (Jonckheere-Terpstra test); $\mathrm{Z}$ = z-statistic (Cochran-Armitage test).

Data from all elective lumbar procedures $(n=2578)$ were pooled. Trends were statistically tested for in a 2-tailed approach. Mean values are presented with standard deviations.

${ }^{*}$ Statistically significant $(p \leq 0.05)$. 
TABLE 5. Subgroup analysis of trends in length of hospital stay and proportion of 1-night hospital stay in the lumbar fusion cohort

\begin{tabular}{|c|c|c|c|c|c|c|c|c|}
\hline \multirow[b]{2}{*}{ Parameter } & \multicolumn{5}{|c|}{ Value } & \multirow[b]{2}{*}{ Trend } & \multirow[b]{2}{*}{ Effect Size } & \multirow{2}{*}{$\begin{array}{l}\text { p Value } \\
\text { (trend) }\end{array}$} \\
\hline & $2013-2014(n=61)$ & $2015(n=48)$ & $2016(n=32)$ & $2017(n=31)$ & $2018(n=27)$ & & & \\
\hline $\begin{array}{l}\text { Length of hospital stay in } \\
\text { hrs, mean }\end{array}$ & $56.9 \pm 28.6$ & $43.3 \pm 16.5$ & $42.2 \pm 17.0$ & $38.6 \pm 9.7$ & $34.9 \pm 7.9$ & Decreasing & $T_{J T}=5331$ & $<0.001^{*}$ \\
\hline 1-night hospital stay, n (\%) & $16(26)$ & $26(54)$ & $20(62)$ & $23(74)$ & $23(85)$ & Increasing & $Z=-5.77$ & $<0.001^{*}$ \\
\hline
\end{tabular}

Data from all patients undergoing ALIF, MI-TLIF, or MI-PLIF $(n=199)$ are included. Trends were statistically tested for in a 2-tailed approach.

${ }^{*}$ Statistically significant $(p \leq 0.05)$.

response sufficiently, the successful implementation of an ERAS program depends on combining a range of key elements in a single perioperative care framework.?

As the number of surgical interventions in the spine keeps increasing over the years, innovation and clinical research has been mainly focused on augmenting surgical techniques and on improving the final, long-term outcomes of interventions. ${ }^{22}$ Still, spine surgery remains known for its often burdensome recovery process. ${ }^{6,24}$ With these opportunities for progress, combined with the influx of evidence supporting the benefits of ERAS in other specialties, such as abdominal and orthopedic surgery, ${ }^{7,10,11}$ the field is preparing for an evolution toward improved perioperative care and short-term outcomes. ${ }^{1,24}$ The first pioneering efforts on ERAS in the spine have emerged in the past years, demonstrating reduced length of stay and acute care costs without increase in readmission rates. ${ }^{2,6,17,23-25}$ Our data provide the first evidence on an ERAS protocol applied to ALIF. Notably, ALIF patients were discharged even earlier than MI-TLIF patients. We also provide corroborating evidence on the safety and efficacy of "fast-track" perioperative care protocols in microdiscectomy, decompression, MI-TLIF, and MI-PLIF, in contrast to a single protocol dedicated to a single technique. This approach better reflects the patient population seen by spine surgeons in daily practice. Further prospec-

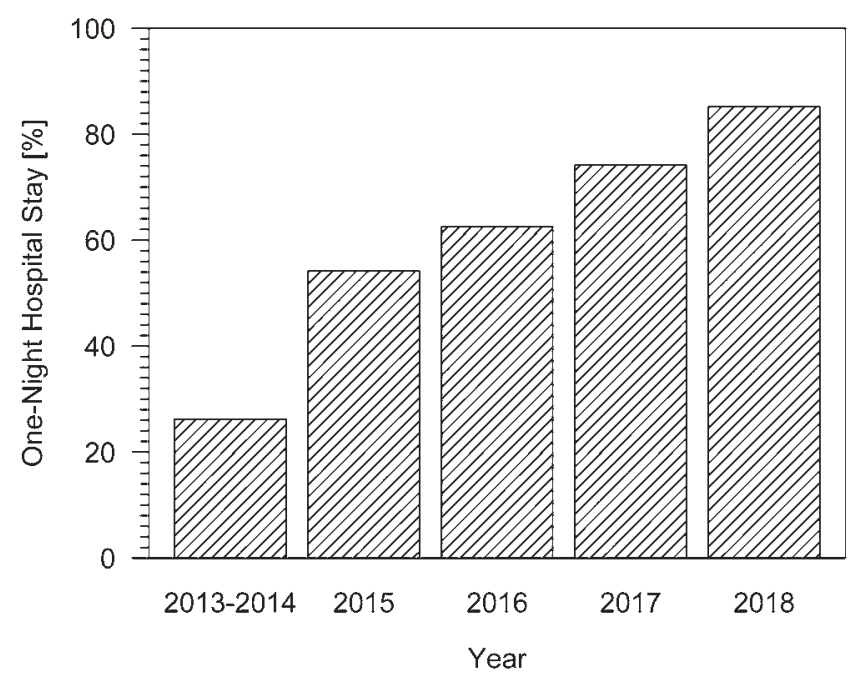

FIG. 2. Proportions of lumbar fusion patients who were discharged the morning after surgery. The trend over the 5-year period since implementation of the protocol, increasing steadily from $26 \%$ in $2013-2014$ to $85 \%$ in 2018 , was statistically significant $(Z=-5.77,2$-tailed $p<0.001)$. tive efforts, such as a randomized controlled trial, are being developed to elevate the evidence on spinal ERAS to a higher level. ${ }^{1}$

Establishing an effective "fast-track" program requires an iterative improvement process. ${ }^{11,24}$ In our 5-year experience, this became evident with the marked increase in patients discharged home early and reduced length of stay, especially in the fusion cohort. In terms of length of hospital stay, we additionally observed a vastly decreasing variance in length of stay over the years. In our prospective registry, data on compliance with single elements of the ERAS protocol were not routinely captured. Although it is conceivable that increased compliance throughout the years greatly influenced the improved perioperative outcomes seen especially in the fusion cohort, we are unable to demonstrate this at present. Nevertheless, there are some factors that in our view contributed most to the improvements. First, there was a conscious decision to move from MI-PLIF to MI-TLIF. This is clearly visible during the transition period from 2013-2014 to 2015 and onwards (Table 2). In line with this transition, preoperative patient education was emphasized and was increasingly focused on preparing the patients for a 1-night hospital stay, if pos-

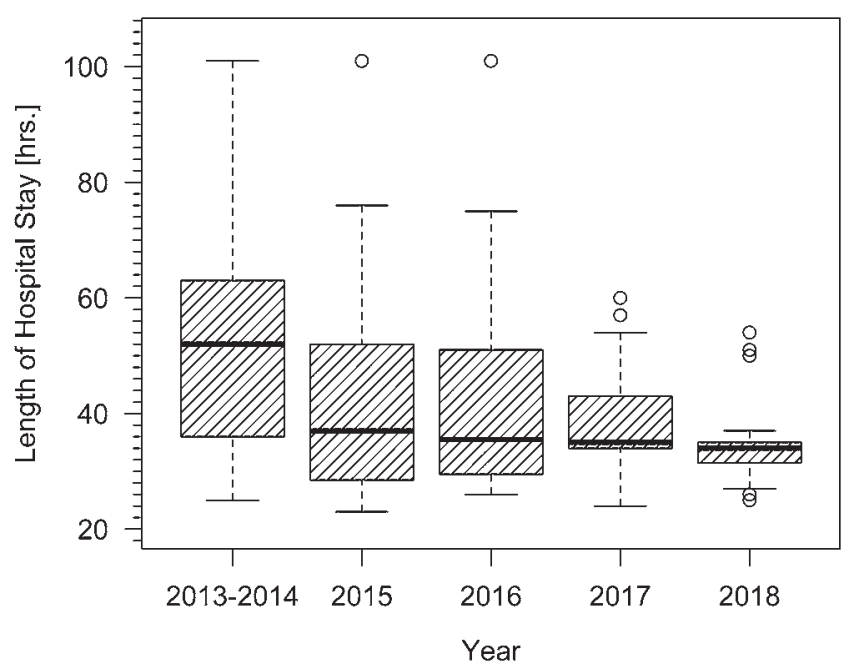

FIG. 3. Length of hospital stay for lumbar fusion patients. The trend over the 5 -year period since implementation of the protocol, decreasing steadily from $2.4 \pm 1.2$ days ( $56.9 \pm 28.6$ hours) in 2013-2014 to $1.5 \pm$ 0.3 days ( $34.9 \pm 7.9$ hours) in 2018 , was statistically significant $\left(T_{J T}=\right.$ 5331 , 2-tailed $p<0.001$ ), with a notable concomitant decrease in variance. Shown are median values (horizontal line), interquartile ranges (boxes), range (whiskers), and extreme outliers (circles). 
sible. During that transitional period, the patient-friendly website, serving as an easily available reference text for patients, was also implemented. Gustafsson et al. found that, within a 5-year period, compliance with an ERAS protocol was elevated from $43 \%$ to $71 \%$ in colorectal surgery? These changes reflect the learning process of all parts of the multidisciplinary ERAS framework. ${ }^{24}$ Proven barriers to implementation are resistance to change, lack of time and staff, poor communication, as well as lack of leadership, coordination, and collaboration. ${ }^{11}$ The initial apprehension of the physician to discharge patients home earlier may also decrease with greater ERAS experience. Implementation of a new protocol may be more complex for a diverse patient population than for patients undergoing a single procedure, with each type of surgery leading to different postoperative symptoms and care requirements. While 30-day and 60-day readmission rates appeared even to decline somewhat, this trend did not reach significance. Our data stress the importance of a dedicated team and associated learning curve. This suggests that the effect size of ERAS interventions will increase when performing an analysis once the initial stages of the implementation process have been passed.

Improved cost-effectiveness of surgical interventions is often a secondary objective when implementing strategies for faster recovery. ${ }^{21,22}$ Wang et al. recently observed that ERAS MI-TLIF led to a reduction in hospitalization costs compared to conventional MI-TLIF. ${ }^{23}$ In 38 ERAS MI-TLIF patients, acute care costs diminished from an average of $\$ 22,656$ to $\$ 19,212$, corresponding to a $15.2 \%$ reduction. In our cohort, nursing costs fell by $46.8 \%$, supporting the cost-effectiveness of "fast-track" protocols in lumbar spine surgery.

Another goal of ERAS protocols lies in reducing both intra- and postoperative adverse events, which have the potential to impair the patients' perioperative well-being and to prolong recovery. Especially in elderly patients, or in those with severe comorbidities, strict patient selection is key to both eligibility for safe ERAS ${ }^{23}$ and to treatment in specialized short-stay clinics that may not have an ICU available. ${ }^{26}$ Administration of prophylactic medication against infections and thrombosis, prevention of hypothermia and fluid imbalance, as well as operative measures that help decrease neurosurgical complications-such as navigation or robotic guidance to reduce the rate of screw malposition-have therefore become integral to successful implementation of ERAS. 4,9,11,12,15,16,18,24

When patients are discharged home early, provisions should be made for them to feel safe, have peace of mind, and most importantly know that the threshold for a call-in, clinical visit, or even readmission is low, in case of any adverse events or uncertainties. In our approach, this includes only minimal behavioral restrictions, and no restrictions in ADL, in order to avoid making patients feel more functionally impaired than they are. All patients have follow-up telephone calls scheduled for 2 days and 14 days after surgery, providing additional opportunities for them to ask questions and increasing their feeling of safety. Moreover, patients can also check the most common FAQs online on the patientfriendly website. From patient feedback, we know that this approach is highly valued. Lastly, questionnaires on satis- faction with the treatment and care process and on PROMs are dispatched to all patients at multiple time points before and after surgery, enabling regular auditing.

In our experience, structured and detailed preoperative counseling is paramount to the implementation of ERAS and especially to the ability to discharge patients home early, safely, and without compromising their comfort. Yi et al. analyzed the influence of a preoperative mindfulness-based stress reduction therapy on postoperative symptoms. ${ }^{27}$ They found that this approach led to a reduction in back pain severity 30 days postoperatively, although they state that their pilot study was not powered to confirm this finding. Our structured preoperative counseling takes around $30 \mathrm{~min}-$ utes and also includes information on how patients should organize transport on the day after surgery, what they may experience in the weeks after surgery, how to integrate their partner or relatives into their postoperative care, information on getting back to work, and how follow-up will be conducted. The core of our preoperative patient education is based on the "Three Golden Rules" as principles of conduct during the recovery process. From our experience, taking ample time for preoperative counseling is crucial for the effectiveness of all other elements in the ERAS protocol.

\section{Limitations}

This is a single-center study, potentially limiting the generalizability of our findings. We were unable to include patients with a BMI greater than 33, age greater than 80 years, or severe comorbidities due to local safety regulations for ambulatory surgery centers. The calculation of cost-effectiveness provided is based on data provided by our Department of Finance, and we are unable to provide absolute cost prices due to regulations set out by the Dutch Authority for Consumers and Markets (ACM). We were unable to determine whether the improvements resulted from improved compliance with the ERAS protocol over the years, as has been previously reported. ? Furthermore, a 5-year period is relatively short for analysis of trends. However, we were highly selective in our statistical analysis and tested trends in a 2-tailed approach, limiting the likelihood of type I errors. We were unable to analyze how comorbidities interact with the efficacy of our protocol. Finally, our study is limited by the lack of a control group.

\section{Conclusions}

Application of an ERAS protocol over 5 years to a diverse population of patients undergoing surgical treatment for degenerative spine conditions, including anterior and posterior lumbar fusion, was safe and effective, without increase in readmissions. Over time and with greater experience, the proportion of patients discharged home early increased markedly. Both adverse events and length of hospital stay are reduced with greater experience with the perioperative care protocol. In fusion procedures, these findings demonstrated an even larger effect size. Prospective controlled or randomized trials are needed to evaluate the efficacy of ERAS approaches in spine surgery on a higher level of evidence.

\section{References}

1. Ali ZS, Ma TS, Ozturk AK, Malhotra NR, Schuster JM, 
Marcotte PJ, et al: Pre-optimization of spinal surgery patients: Development of a neurosurgical enhanced recovery after surgery (ERAS) protocol. Clin Neurol Neurosurg 164:142-153, 2018

2. Chang HK, Kolcun JPG, Chang PY, Wang MY: Enhanced Recovery After Surgery ${ }^{\mathrm{TM}}$ awake minimally-invasive transforaminal lumbar interbody fusion: 2-dimensional operative video. Oper Neurosurg (Hagerstown) [epub ahead of print], 2018 [Erratum in Oper Neurosurg (Hagerstown) 15:610, 2018]

3. Clark AJ, Safaee MM, Khan NR, Brown MT, Foley KT: Tubular microdiscectomy: techniques, complication avoidance, and review of the literature. Neurosurg Focus 43(2):E7, 2017

4. Fearon $\mathrm{KCH}$, Ljungqvist $\mathrm{O}$, Von Meyenfeldt $\mathrm{M}$, Revhaug A, Dejong CHC, Lassen K, et al: Enhanced recovery after surgery: a consensus review of clinical care for patients undergoing colonic resection. Clin Nutr 24:466-477, 2005

5. Goldstein CL, Macwan K, Sundararajan K, Rampersaud YR: Perioperative outcomes and adverse events of minimally invasive versus open posterior lumbar fusion: meta-analysis and systematic review. J Neurosurg Spine 24:416-427, 2016

6. Grasu RM, Cata JP, Dang AQ, Tatsui CE, Rhines LD, Hagan $\mathrm{KB}$, et al: Implementation of an Enhanced Recovery After Spine Surgery program at a large cancer center: a preliminary analysis. J Neurosurg Spine 29:588-598, 2018

7. Gustafsson UO, Hausel J, Thorell A, Ljungqvist O, Soop M, Nygren J: Adherence to the enhanced recovery after surgery protocol and outcomes after colorectal cancer surgery. Arch Surg 146:571-577, 2011

8. Kehlet H: Multimodal approach to control postoperative pathophysiology and rehabilitation. Br J Anaesth 78:606617, 1997

9. Lassen K, Soop M, Nygren J, Cox PBW, Hendry PO, Spies $\mathrm{C}$, et al: Consensus review of optimal perioperative care in colorectal surgery: Enhanced Recovery After Surgery (ERAS) Group recommendations. Arch Surg 144:961-969, 2009

10. Liu VX, Rosas E, Hwang J, Cain E, Foss-Durant A, Clopp M, et al: Enhanced recovery after surgery program implementation in 2 surgical populations in an integrated health care delivery system. JAMA Surg 152:e171032, 2017

11. Ljungqvist O, Scott M, Fearon KC: Enhanced recovery after surgery: a review. JAMA Surg 152:292-298, 2017

12. Molliqaj G, Schatlo B, Alaid A, Solomiichuk V, Rohde V, Schaller K, et al: Accuracy of robot-guided versus freehand fluoroscopy-assisted pedicle screw insertion in thoracolumbar spinal surgery. Neurosurg Focus 42(5):E14, 2017

13. Rushton A, Eveleigh G, Petherick E-J, Heneghan N, Bennett R, James G, et al: Physiotherapy rehabilitation following lumbar spinal fusion: a systematic review and meta-analysis of randomised controlled trials. BMJ Open 2:e000829, 2012

14. Schröder ML, de Wispelaere MP, Staartjes VE: Are patientreported outcome measures biased by method of follow-up? Evaluating paper-based and digital follow-up after lumbar fusion surgery. Spine J 19:65-70, 2019

15. Schröder ML, Staartjes VE: Revisions for screw malposition and clinical outcomes after robot-guided lumbar fusion for spondylolisthesis. Neurosurg Focus 42(5):E12, 2017

16. Schroerlucke SR, Wang MY, Cannestra AF, Good CR, Lim $\mathrm{J}, \mathrm{Hsu} \mathrm{VW}$, et al: Complication rate in robotic-guided vs fluoro-guided minimally invasive spinal fusion surgery: report from MIS refresh prospective comparative study. Spine J 17:S254-S255, 2017

17. Soffin EM, Vaishnav AS, Wetmore D, Barber L, Hill P, Gang $\mathrm{CH}$, et al: Design and implementation of an enhanced recovery after surgery (ERAS) program for minimally invasive lumbar decompression spine surgery: initial experience. Spine (Phila Pa 1976) [epub ahead of print], 2018

18. Solomiichuk V, Fleischhammer J, Molliqaj G, Warda J, Alaid A, von Eckardstein K, et al: Robotic versus fluoroscopy-guided pedicle screw insertion for metastatic spinal disease: a matchedcohort comparison. Neurosurg Focus 42(5):E13, 2017
19. Staartjes VE, Vergroesen PA, Zeilstra DJ, Schröder ML: Identifying subsets of patients with single-level degenerative disc disease for lumbar fusion: the value of prognostic tests in surgical decision making. Spine J 18:558-566, 2018

20. Streitberg B, Röhmel J: Exact distributions for permutation and rank tests: an introduction to some recently published algorithms. Stat Softw Newsl 12:10-17, 1986

21. Twitchell S, Karsy M, Reese J, Guan J, Couldwell WT, Dailey A, et al: Assessment of cost drivers and cost variation for lumbar interbody fusion procedures using the Value Driven Outcomes database. Neurosurg Focus 44(5):E10, 2018

22. Wainwright TW, Immins T, Middleton RG: Enhanced recovery after surgery (ERAS) and its applicability for major spine surgery. Best Pract Res Clin Anaesthesiol 30:91-102, 2016

23. Wang MY, Chang HK, Grossman J: Reduced acute care costs with the ERAS ${ }^{\circledR}$ minimally invasive transforaminal lumbar interbody fusion compared with conventional minimally invasive transforaminal lumbar interbody fusion. Neurosurgery 83:827-834, 2018

24. Wang MY, Chang PY, Grossman J: Development of an enhanced recovery after surgery (ERAS) approach for lumbar spinal fusion. J Neurosurg Spine 26:411-418, 2017

25. Wang MY, Grossman J: Endoscopic minimally invasive transforaminal interbody fusion without general anesthesia: initial clinical experience with 1-year follow-up. Neurosurg Focus 40(2):E13, 2016

26. Wang MY, Widi G, Levi AD: The safety profile of lumbar spinal surgery in elderly patients 85 years and older. Neurosurg Focus 39(4):E3, 2015

27. Yi JL, Porucznik CA, Gren LH, Guan J, Joyce E, Brodke DS, et al: The impact of preoperative mindfulness-based stress reduction on postoperative patient-reported pain, disability, quality of life, and prescription opioid use in lumbar spine degenerative disease: a pilot study. World Neurosurg 121:e786-e791, 2019

\section{Disclosures}

The authors report no conflict of interest concerning the materials or methods used in this study or the findings specified in this paper.

\section{Author Contributions}

Conception and design: Staartjes, Schröder. Acquisition of data: de Wispelaere, Schröder. Analysis and interpretation of data: Staartjes, Schröder. Drafting the article: Staartjes, Schröder. Critically revising the article: all authors. Reviewed submitted version of manuscript: all authors. Approved the final version of the manuscript on behalf of all authors: Staartjes. Statistical analysis: Staartjes. Administrative/technical/material support: de Wispelaere, Schröder. Study supervision: Schröder.

\section{Supplemental Information}

\section{Online-Only Content}

Supplemental material is available online.

The $\mathrm{R}$ code was used for the statistical analysis and rendering of the figures. The code was executed in R Version 3.5.1 (The $\mathrm{R}$ Foundation for Statistical Computing) on a machine running Windows 10 (Microsoft Corp.). The raw data will be made available by the authors on request.

$R$ code. https://thejns.org/doi/suppl/10.3171/2019.1.FOCUS 18646.

Supplementary Tables 1-3. https://thejns.org/doi/suppl/10. 3171/2019.1.FOCUS18646.

\section{Correspondence}

Victor E. Staartjes: Bergman Clinics, Amsterdam, The Netherlands.victor.staartjes@gmail.com. 